\title{
Evaluation of Antiparkinson Activity of Hesperetin \& Pyridoxine against Olanzepine \& Manganese Induced Parkinsonism
}

\author{
Yogita Dobhal" and Eakta Kandpal
}

Department of Pharmaceutical Sciences, Sardar Bhagwan Singh PG Institute of Biomedical Sciences \& Research, Balawala, Dehradun, Uttarakhand, India.

*Corresponding author: Yogita Dobhal, Department of Pharmaceutical Sciences, Sardar Bhagwan Singh PG Institute of Biomedical Sciences \& Research, Balawala, Dehradun, Uttarakhand, India, Tel: +91 09410790829; Email: yogita_sharma05@ rediffmail.com

Citation: Dobhal Y (2017) Evaluation of Antiparkinson Activity of Hesperetin \& Pyridoxine against Olanzepine \& Manganese Induced Parkinsonism. J Pharma Pharma Sci 2017: G 109. DOI:10.29011/2574-7711/100009

Revived Date: 7 November 2016; Accepted Date: 11 February 2017; Published Date: 18 February 2017

\section{Abstract}

Objective: The present study was aimed to evaluate antiparkinson activity of hesperetin and pyridoxine against olanzepine and manganese induced Parkinsonism rat model.

Material and Methods: Olanzepine $(20 \mathrm{mg} / \mathrm{kg})$ and manganese $(100 \mathrm{mg} / \mathrm{kg})$ were used for the induction of Parkinsonism for 28 days. Test groups receive simultaneous treatment of hesperitin $(10 \mathrm{mg} / \mathrm{kg})$ and pyridoxine $(10 \mathrm{mg} / \mathrm{kg})$. Then the level of enzymes viz. LPO, SOD, GSH, catalase and level of neurotransmitters such as dopamine and acetylcholine were evaluated. Selegiline $(30 \mathrm{mg} / \mathrm{kg})$ was used as standard drug in reference to the vehicle control and test groups.

Results: Among all the test groups (T1, T2 \& T3), group T3 maintain the level of enzymes (LPO, SOD, GSH, Catalase) and neurotransmitters (Dopamine and Acetylcholine) statistically significant as compared to vehicle control group.

Conclusion: From the results of the present study hesperetin and pyridoxine may act as potential antioxidants in treatment of Parkinsonism

Keywords: Parkinsonism, Hesperetin, Pyridoxine, Antioxidants.

\section{Introduction}

Parkinsonism is 2 nd commonest neurodegenerative disease in the world. It was estimated that the number of people suffering with it will rise from 4.1 to 4.6 million in 2020. In Asia there are expected to increase the Parkinsonism patients from 2.757 million in 2005 to 6.17 million in 2030 [1]. It is neurodegenerative disease in which degeneration of dopaminergic neuron in the substantial nigra of brain and characterized by movement disorders like rigidity, bradikinesia, postural instability, tremors [2]. Progressive loss of dopamine containing neuron is common signs of aging so it is started commonly in the age of 60 . In the early stage of disease the face may show less expression and arms may not swing when walk and speech may become soft or slurred. In this stage there is about $80 \%$ loss of dopamine and lead to Parkinsonism [3]. Catabolism is the main Mechanism for deactivation of Dopamine. It involves multiple pathways like oxidative deamination by MAO and COMT and conjugation by sulfotransferase and glucoronidase [4]. Energy metabolism, oxidative stress, chronic infection, programmed cell death, neurotoxins and excitotoxicity are also responsible for Parkinsonism [5].

From the literature review it was found that flavonoids, tannins and phenolic constituents from plant origin are potential antioxidants and play an essential role in the prevention of neurodegenerative diseases. A direct relationship between antioxidant activity and phenolic content of plant extracts has been reported. In recent years, there has been increasing interest in investigating polyphenols from botanical source for possible neuroprotective effects against neurodegenerative disease. Antioxidant activity of flavonoids is attributed to their hydrogen-donating ability. Indeed, the phenolic groups of flavonoids serve as a source of a readily available " $\mathrm{H}$ " atoms such that the subsequent radicals produced 


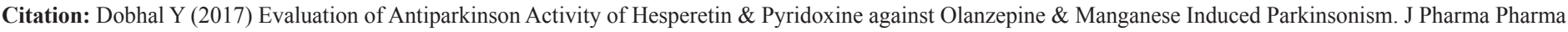
Sci 2017: G 109

can be delocalized over the flavonoids structure. Therefore, the present study was aimed to evaluate antiparkinson activity of pyridoxine and hesperetin against olanzepine and manganese induced Parkinsonism rat model $[6,7]$.

\section{Materials and Methods}

\section{Equipments and chemicals}

The equipments were used from Shimadzu, Narang Scientific Works Pvt. Ltd., Inco Ambala, Remi motors Ltd. Mumbai, Olyumpus. All chemicals were analytical grades used in the study and purchased from RANKEM; MERCK; CDH; LOBA CHEM. Drugs were used in the study such as Selegilline (HIMEDIA), Olanzepine (SIGMA ALDRICH), Hesperitine (TCI).

\section{Animals}

Westar albino rats of body weight 150-250 gm and 6month of age of either used for study. The animals were housed in standard polypropylene cages and maintained under controlled room temperature $(22 \pm 2 \mathrm{oc})$ and humidity $(55 \pm 5 \%)$ with $12: 12$ hour light and dark cycle. All the animals were provided with commercially available rat normal pallet diet and water ad labium. The guidelines of the committee for the purpose of control and supervision of experiment on Animals (CPCSEA) of India was followed and prior permission for granted by the institutional Animal Ethics Committee (Reg No. 273/CPCSEA) for conducting the animal experimental studies.

\section{Protocol}

Albino Wister rats are divided into six groups and each group had five animals. The study protocol was carried out for 28 days. Inducer drugs were Manganese $(100 \mathrm{mg} / \mathrm{kg})$ and Olanzepine $(20 \mathrm{mg} / \mathrm{kg})$.

Group 1: (Normal; $\mathrm{n}=5$ ) Animals received normal saline

Group 2: (Control; n=5) Manganese $(100 \mathrm{mg} / \mathrm{kg})$ and Olanzepine $(20 \mathrm{mg} / \mathrm{kg})$

Group 3: (Standard; $n=5)$ Inducer drug with Selegilline (30mg/ $\mathrm{kg})$

\begin{tabular}{|c|c|c|c|c|c|c|}
\hline Groups & DA Level & Ach Level & LPO Level & SOD Level & $\begin{array}{c}\text { Reduced glutathi- } \\
\text { one Level }\end{array}$ & $\begin{array}{c}\text { Enzyme Catalase } \\
\text { Level }\end{array}$ \\
\hline Normal & $1.25 \pm 0.44$ & $19.56 \pm 2.85$ & $54.33 \pm 10.39$ & $64.33 \pm 10.39$ & $66.72 \pm 13.05$ & $0.333 \pm 0.33$ \\
\hline Control & $5.75 \pm 034$ & $65.14 \pm 2.51$ & $84.31 \pm 0.39$ & $55.53 \pm 35.33$ & $90.03 \pm 35.35$ & $0.11 \pm 0.79$ \\
\hline Standard & $1.59 \pm 0.35$ & $23.05 \pm 0.78$ & $74.33 \pm 87.39$ & $77.36 \pm 2.68$ & $68.08 \pm 15.35$ & $1.766 \pm 0.26$ \\
\hline T1 & $5.47 \pm 0.25$ & $66.79 \pm 3.53$ & $94.32 \pm 70.87$ & $67.07 \pm 0.36$ & $202.29 \pm 23.24$ & $7.166 \pm 2.72$ \\
\hline T2 & $5.00 \pm 0.08$ & $59.35 \pm 2.70$ & $94.34 \pm 10.39$ & $71.58 \pm 0.48$ & $202.57 \pm 32.37$ & $7.895 \pm 0.68$ \\
\hline T3 & $5.22 \pm 0.43$ & $57.44 \pm 2.89$ & $90.33 \pm 12.89$ & $76.09 \pm 2.04$ & $196.31 \pm 3.18$ & $10.165 \pm 0.87$ \\
\hline
\end{tabular}

Table 1: Neurotransmitters level and biochemical analysis.
Group 4: (Treatment Group-T1; $n=5$ ) Inducer drug with Pyridoxine $(10 \mathrm{mg} / \mathrm{kg})$

Group 5: (Treatment Group-T2; n=5) Inducer drug with Hespere$\operatorname{tin}(10 \mathrm{mg} / \mathrm{kg})$

Group6: (Treatment Group-T3; n=5) Inducer drug with Hesperetin $(10 \mathrm{mg} / \mathrm{kg})+$ Pyridoxine $(10 \mathrm{mg} / \mathrm{kg})$

\section{Estimation of antioxidant \& neurotransmitter level}

Animal was fasted overnight and brains are removed quickly and placed in ice cold saline and are quickly dissected out on a Petridis chilled on crushed ice. The tissue are weighed and homogenized in 0.1M Phosphate buffer (PH 7.2). The homogenate was centrifuged at $3000 \mathrm{rpm}$ for $10 \mathrm{~min}$ and resultant cloudy supernatant liquid was used for biochemical estimation. These biochemical parameters were Reduced glutathione [8], Lipid per oxidation [9], Catalase [10]. Superoxide have ability to inhibit auto-oxidation of epinephrine to adenochrome at PH 10.2. This inhibition can be measured spectrophotometer at $480 \mathrm{~nm}$. The radio immune essay was developed by Solomon A. Berson and Rosalyn S. in 1950. It is a technique for measurement of the level of neurotransmitters.

\section{Results}

In the present study Parkinsonism was induced by administration of manganese $(100 \mathrm{mg} / \mathrm{kg})$ and olanzepine $(20 \mathrm{mg} / \mathrm{kg})$ and shown decreased level of dopamine, superoxide dismutase, reduced glutathione and enzyme catalase while increased level of acetylcholine and lipid peroxide in control group as compared to the normal vehicle group. In standard group after administration of selegiline $(30 \mathrm{mg} / \mathrm{kg})$ there was marked rise in level of dopamine, superoxide dismutase, reduced glutathione and enzyme catalase where as decreased level of acetylcholine and lipid peroxide as compared to the control group. Furthermore, in treatment groups T1, T2, T3 were shown significant increased level of protecting neurotransmitters and enzymes after treatment. Moreover, in treatment group T3 among all the groups resulted statically significant increased level of dopamine, superoxide dismutase, reduced glutathione and enzyme catalase and decreased level of acetylcholine and lipid peroxide 


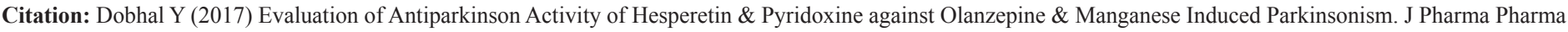
Sci 2017: G 109

The statistical significance of difference between means was calculated by ANNOVA followed by Dennett's post hoc test. Values are expressed as Mean \pm SEM; $n=5$ in each group. DA-dopamine; Ach-acetylcholine; LPO-lipid peroxide; SOD-superoxide dismutase.

\section{Discussion}

Parkinson's develops gradually and starting with a tremor in just one hand, but while tremor may be well known sign of Parkinson's disease. The disorder also commonly causes stiffness or slowing of movement. In the early stage of disease the face may show less expression and arms may not swing when walk and speech may become soft or slurred. In the Parkinsonism presence of protein alpha-syncline, which are also called lewy bodies are founds in mid brain, brain stem and olfactory bulb. These area of brain correlate to non motor function such as smell and sleep regulation and these functions are also disturbed in Parkinsonism [11].

The antioxidants have ability to decrease the oxidative stress which is one of the significant approaches to overcome the symptoms of Parkinson's. Hesperetin is natural falconoid and has antioxidant properties. Flavonoids are group of polyphenolic compounds of low molecular weight $(200-600 \mathrm{~g} / \mathrm{mol})$. They have various pharmacological properties and act as scavengers of free radicals by $\mathrm{OH}$ groups in their molecular structure [12]. Several studies have reported that hesperetin have anti-inflammatory, antioxidant, anti-carcinogenic, and neuroprotective effects. Hesperetin is a compound with 3 hydroxyl groups that maintain greater antioxidant potency and ability to activate cellular antioxidant preventing enzymes [13, 14]. Pyridoxine (Vitamin B6) is required for the synthesis of neurotransmitters and myelin formation. Several activities such as anti-oxidant, anti-thyroid, anti-inflammatory, ant autism etc. were reported in the literature $[15,16]$.

The present study was aimed to evaluate antiparkinson activity of pyridoxine and hesperetin against olanzepine and manganese induced Parkinsonism rat model. The olanzepine is an antipsychotic drug and cause parkinsonism like symptoms in long term use, it binds transiently to D2 receptor this transient binding is sufficient for antipsychotic effects to take place but they also inhibit the natural present dopamine thus produce parkinsonism. Manganese is heavy metal and cause neurotoxicity. It decreases the concentration of dopamine in the dopaminergic neuron and due to low level of dopamine in the brain generation of Parkinsonism like symptoms. Selegiline $(30 \mathrm{mg} / \mathrm{kg})$ administered as a standard drug and it was found that the level of dopamine, reduced glutathione, superoxide dismutase and enzyme catalase were significantly increased while level of acetylcholine and LPO were decreased as compared to control group. In the treatment groups viz. T1, T2, $\mathrm{T} 3$ according to the protocol pyridoxine $(10 \mathrm{mg} / \mathrm{kg})$ and hesperetin $(10 \mathrm{mg} / \mathrm{kg})$ individually (T1 and $\mathrm{T} 2)$ and in combination of both
(T3) were administered orally for 28 days. The blood samples were withdrawn to evaluate different parameters such as level of enzymes and level of neurotransmitters. Furthermore, among all these treatment groups the $\mathrm{T} 3$ group shown statically significant results as compared to control group in terms of increased level of dopamine, reduced glutathione, superoxide dismutase and enzyme catalase while level of acetylcholine and lipid peroxides were decreased.

\section{Conclusion}

The antioxidants have ability to decrease the oxidative stress which is one of the significant approaches to overcome the symptoms of Parkinsonism. So the combination of hesperetin and pyridoxine may be one of the beneficial approaches in the treatment of Parkinsonism.

\section{References}

1. Ballard PA, Tetrud JW, Langston JW (1985) Permanent human Parkinsonism due to 1-methyl-4-phenyl-1, 2, 3, 6-tetrahydropyridine (MPTP): seven cases. Neurology 35: 949-56.

2. Albin RL, Reiner A, Anderson KD, Dure LS, Handelin B, et al. (1992) Preferential loss of striatum-external pallidal projection neurons in presymptomatic Huntington's disease. Ann. Neurol 31: 425-423.

3. Chatellier G, Lacomblez L (1990) Tacrine (tetrahydroaminoacridine; THA) and lecithin in senile dementia of the Alzheimer type: A Tetrahydromaminoacridine. B.M.J 300: 495-499.

4. Friedman JH, Factor SA (2000) A typical antipsychotics in the treatment of drug-induced psychosis in Parkinson's disease 15: 201-211.

5. Chase TN (1997) Levodopa therapy: Consequences of no physiologic replacement of dopamine. Neurology 50: S17-S25.

6. Anna K. Jäger, Lasse Saaby (2011) Flavonoid and the CNS. Molecules 16: 1471-1485.

7. Hollman, PCH (2004) Absorption, Bioavailability, and Metabolism of Flavonoids. Arch. Physiol. Biochem. 42: 74-83.

8. Maria S Moron, Joseph W, Depierre, Bengt Mannervik (1979) Levels of glutathione, glutathione reductase and glutathione S-transferase activities in rat lung and liver. Biochimica et Biophysica Acta (BBA) General Subjects 582: 67-78.

9. Janero DR (1990) Malondialdehyde and thiobarbituric acid-reactivity as diagnostic indices of lipid per oxidation and per oxidative tissue injury. Free Radic. Biol. Med 9: 515-540.

10. Brannan TS, Maker HO, Raess IP (1981) Regional distribution of catalyse in adult rat brain. J. Neurochem 86: 307- 309.

11. Rang H, Dale M, Ritter J, Moore P (2007) Pharmacology, 6th ed; Churchill Livingstone: Edinburgh, UK.

12. Billett EE (2004) Monoamine oxidase (MAO) in human peripheral tissues. Neurotoxicology 25: 139-148.

13. Selcuk Kara, Baran Gencer, Turan Karaca, Hasan Ali Tufan, Sedat Arikan, et al (2014) Protective effect of hesperetin and naringenin against apoptosis in ischemia induced retinal injury in rats. Sci. World J 97: 824. 


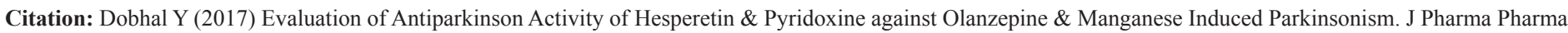
Sci 2017: G 109

14. Majumdar S, Srirangam R (2009) Solubility, stability, physicochemical characteristics and in vitro ocular tissue permeability of hesperidins: a natural bioflavonoid. Pharm. Res 26: 1217-1225.

15. Mousain-Bosc M1, Roche M, Polge A, Pradal-Prat D, Rapin J, et al. (2006) Improvement of neurobehavioral disorders in children supplemented with magnesium-vitamin B6. I. Attention deficit hyperactivity disorders. Magnesium Research 19: 46-52.

16. Malouf R, Grimley Evans J (2003) The effect of vitamin B6 on cognition. Cochrane Database Syst. Rev 4: CD004393. 